\title{
Development and initial validation of the Cardiovascular Disease Acceptance and Action Questionnaire (CVD-AAQ) in an Italian sample of cardiac patients
}

\section{Chiara A. M. Spatola ${ }^{1,2}$ *, Emanuele A. M. Cappella ${ }^{1,2}$, Christina L. Goodwin ${ }^{3}$, Matteo Baruffi ${ }^{1,2}$, Gabriella Malfatto ${ }^{4}$, Mario Facchini ${ }^{4}$, Gianluca Castelnuovo ${ }^{1,2}$, Gian Mauro Manzoni ${ }^{1,5}$ and Enrico Molinari ${ }^{1,2}$}

\author{
${ }^{1}$ Psychology Research Laboratory, Istituto Auxologico Italiano - Istituto di Ricovero e Cura a Carattere Scientifico, Milan, Italy \\ 2 Department of Psychology, Catholic University of Milan, Milan, Italy \\ ${ }^{3}$ The Ohio State University, Columbus, OH, USA \\ ${ }^{4}$ Cardiology Division, Istituto Auxologico Italiano - Istituto di Ricovero e Cura a Carattere Scientifico, Milan, Italy \\ ${ }^{5}$ Faculty of Psychology, eCampus University, Novedrate, Como, Italy
}

Edited by:

Victoria Vaughan Dickson, New York University, USA

\section{Reviewed by:}

Marco Innamorati, Università Europea di Roma, Italy

Emily Martin, MD Anderson Cancer

Center, USA

\section{*Correspondence:}

Chiara A. M. Spatola, Psychology

Research Laboratory, Istituto

Auxologico Italiano - Istituto di

Ricovero e Cura a Carattere

Scientifico, Milan, Italy

e-mail: c.spatola@auxologico.it
Psychological inflexibility refers to the attempt to decrease internal distress even when doing so is inconsistent with life values, and has been identified as a potential barrier to making and maintaining health behavior changes that are consistent with a heart-healthy lifestyle. Disease- and behavior-specific measures of psychological inflexibility have been developed and utilized in treatment research. However, no specific measure has been created for patients with heart disease. Thus, the CardioVascular Disease Acceptance and Action Questionnaire (CVD-AAQ) was developed. The present study is aimed to evaluate the psychometric properties of the CVD-AAQ and to explore its association with measures of psychological adjustment and cardiovascular risk factors in an Italian sample of 275 cardiac patients. Exploratory factor analysis showed a structural one-factor solution with satisfactory internal consistency and test-retest reliability. The relation with other measures was in the expected direction with stronger correlations for the theoretically consistent variables, supporting convergent and divergent validity. CVD-AAO scores were associated with general psychological inflexibility, anxiety and depression and inversely correlated with psychological well-being. Moreover, the results showed that CVD-AAQ scores are associated with two relevant risk factors for cardiac patients, namely low adherence to medication and being overweight. In sum, results suggest that the CVD-AAQ is a reliable and valid measure of heart disease-specific psychological inflexibility with interesting clinical applications for secondary prevention care.

Keywords: psychological inflexibility, acceptance, cardiovascular disease, adherence to treatment, experiential avoidance, Cardiovascular Disease Acceptance and Action Questionnaire

\section{INTRODUCTION}

A large portion of cardiovascular risk can be attributed to lifestyle factors, such as unhealthy diet, low physical activity, and smoking (Yusuf etal., 2004). Although the reduction of these unhealthy habits has resulted in a significant decline in mortality rate comparable to that shown by cardio-preventive pharmacotherapy, few patients successfully improve their negative health-behaviors and even fewer maintain acquired changes in the long-term (Dorneleas, 2008). Moreover, high non-adherence rates to cardio-protective medications and subsequent adverse outcomes have been well documented (Ho et al., 2008). Current research has demonstrated that difficulties with behavior change may be related to a lack of psychological flexibility, a concept targeted in acceptance and commitment therapy (ACT; Hayes et al., 1999).

Psychological flexibility is defined as the ability to be fully present in each moment which includes accepting one's own internal experiences even when unpleasant and distressful, and willingly engaging in behaviors that are consistent with one's life values (Hayes et al., 2006).

Two major concepts are involved in the definition of psychological flexibility: the present moment contact with one's thoughts, emotions, and bodily sensations (experiential acceptance), and the importance of maintaining values-driven behaviors. In contrast, psychological inflexibility is the attempt to decrease cognitive and physical distress even when doing so may sacrifice long-term behavioral goals (Luoma et al., 2011).

Since adopting and maintaining a healthy lifestyle often entails distressing internal experiences, individuals with a low ability to tolerate internal distress might be less likely to adopt and maintain behavioral changes. Previous research evaluating adherence to health-behaviors note the importance of distress tolerance; for example, changes in diet often require compromises in portionsize and taste (Forman et al., 2007), exercise is associated with physical discomfort and exercise-anxiety (Butryn etal., 2011), and abstaining from nicotine is associated with cravings (Gifford 
et al., 2004; Brown et al., 2005). The relevant role of psychological inflexibility in preventing behavior change is confirmed by recent studies, showing the promise of acceptance-based interventions at increasing physical activity (Butryn et al., 2011), the ability of managing food cravings (Forman et al., 2007), and weight loss maintenance (Forman et al., 2009).

Moreover, variables found to be impacted by psychological inflexibility, such as depression, anxiety and stress have been shown to negatively impact cardiovascular risk and prognosis (Januzzi et al., 2000; Rozanski et al., 2005; Dimsdale, 2008; Rothenbacher et al., 2014) through the mediation of biological mechanisms such as hypertension and blood pressure reactivity to stress, (Strike and Steptoe, 2004) and behavioral pathways such as lower medication adherence and lesser likelihood to adopt healthy behaviors (Rothenbacher et al., 2014).

Psychological inflexibility is also inversely associated with quality of life, perceived health, and positive emotional experiences (Bond et al., 2011; Gaudiano, 2011). Thus, it is important to investigate the relationship between psychological inflexibility, mental health, quality of life, and the development and progression of coronary heart disease. Understanding such associations will be especially useful in the context of secondary prevention efforts, such as cardiac rehabilitation.

While general measures of psychological inflexibility do exist, to date no instrument has been validated for reliably measuring cardiovascular disease-specific psychological inflexibility. The most established measure of general psychological flexibility is the Acceptance and Action Questionnaire (AAQ; Hayes et al., 2004), which cuts across diagnostic categories and psychological problems. Research on psychological inflexibility in many different samples has shown that the AAQ scores predict $16-25 \%$ of the variance in outcomes across a broad range of psychological health conditions (Hayes et al., 2006). However, versions with a specific focus may offer greater precision in the measurement of psychological flexibility within particular contexts or populations. For example, acceptance of pain as measured by the Chronic Pain Acceptance Questionnaire (CPAQ) appears to partially mediate the relationship between pain severity, and the degree of pain interference and emotional distress (Fish et al., 2010). Similarly, body image flexibility improved prediction of disordered eating, above and beyond overall psychological flexibility even after controlling for body image dissatisfaction and BMI (Sandoz et al., 2013). Comparable results have been shown for other specific variants of the $\mathrm{AAQ}$ and related measures of psychological flexibility in such areas as obesity (Lillis et al., 2009), diabetes (Gregg et al., 2007), epilepsy (Lundgren et al., 2008), tinnitus (Westin et al., 2008), substance abuse (Luoma et al., 2011) and smoking (Gifford et al., 2004).

These results provide support for the usefulness of developing measures of psychological flexibility that are domain specific.

Cardiovascular disease is a disease that requires adjustment to both change in lifestyle and change in self-conception (i.e., identification as a cardiovascular patient). This change in selfconception can be difficult and potentially harmful if the person with heart disease becomes distressed as a result of this new selfconception and begins to avoid disease-specific thoughts, feelings, and behaviors. Behavior-specific measures related to the adoption of healthy lifestyles, such as the Food AAQ (Juarascio et al., 2011) or the Physical Activity AAQ (Butryn et al., 2014) could be usefully adopted with cardiac patients. However, their use will provide information about those behaviors independent of heart disease, which is less helpful when attempting to alter behavior in the context of cardiovascular disease and a changing self-conception. Lastly, a single disease-specific measure will require less time from patients and participants than multiple behavior-specific measures, thus reducing overall patient burden.

Therefore the CardioVascular Disease AAQ (CVD-AAQ) was created to assess patient tendency to avoid difficult disease-related thoughts and feelings and the consequent lack of willingness to engage in recommended life-style changes.

The aim of the present study is threefold:

(1) to evaluate the psychometric properties of CVD-AAQ;

(2) to explore the association between patient demographics and CVD-AAQ scores;

(3) to evaluate the association between CVD-AAQ scores, rates of medication adherence and BMI in a sample of cardiac patients.

\section{MATERIALS AND METHODS PARTICIPANTS}

This study involved 275 participants who were receiving outpatient cardiac rehabilitation.

Demographic information is summarized in Table 1. The majority of participants were married, completed high school, and retired from the workforce.

\section{MEASURES}

General and disease-specific psychological infexibility

The $C V D-A A Q$ is an adaptation of a more general measure of experiential acceptance and avoidance, the AAQ (Bond

Table 1 | Demographic characteristics of the sample.

\section{Demographic variables}

Mean age (years) $\quad 65.50$

Gender ( $\%$ female) $\quad 21.80$

Marital status (\%)

Never married

10.20

Married

68.70

Separated

5.10

Divorced

5.10

Widowed

10.90

\section{Education (\%)}

Elementary school

18.20

Junior high school

25.50

High school

40.70

College education

15.60

\section{Employment (\%)}

Employed

32.00

Retired

57.50

Housewives

5.10

Unemployed
5.50 
et al., 2011). The CVD-AAQ was created to measure individual acceptance of thoughts and feelings related to cardiovascular illness and the degree to which such thoughts and feelings interfere with valued action. The CVD-AAQ items were developed by two of the authors (CAMS and CLG) who had prior experience with both third generation cognitive behavioral treatments and cardiac rehabilitation patients. The CVD-AAQ initial items pool contained 10-items (e.g., I avoid thinking about the risks I face if I don't take care of my heart) rated on a 7-point Likert-type scale ranging from 1 (never true) to 7 (always true). High scores represent high psychological inflexibility.

General psychological inflexibility was measured using the AAQ-II (Bond et al., 2011) a seven-item measure of psychological inflexibility and experiential avoidance. High scores represent more psychological inflexibility. The Italian version of the questionnaire has shown good reliability $(\alpha=0.83)$ and high convergent validity (Pennato et al., 2013). Cronbach's alpha for the current study is 0.75 .

\section{General psychological well-being}

Psychological health was measured by the Psychological General Well-Being (PGWB) Inventory (Dupuy, 1984), validated in Italian (Grossi et al., 2002). Six affective states are assessed within six subscales: anxiety, depressed mood, positive well-being, self-control, general health, and vitality. For each subscale higher scores reflect higher levels of well-being. In the current study PGWB Cronbach's alpha was 0.94 for the total score, 0.86 for anxiety, 0.83 for depressed mood, 0.67 for self-control, 0.83 for positive well-being, 0.59 for general health, and 0.81 for vitality.

\section{Psychological stress}

Psychological stress was measured using the 10-item Perceived Stress Scale, (PSS-10; Cohen et al., 1983), assessing the degree to which situations in one's life are appraised as stressful. For the current study, the scale was translated to Italian. The PSS-10 has shown good validity and internal reliability ranging from 0.78 to 0.91 (Cohen and Janicki-Deverts, 2012). Cronbach's alpha for this study is 0.83 .

\section{Self-efficacy}

The Generalized Self-Efficacy Scale (GSE; Schwarzer and Jerusalem, 1995), was utilized as a measure of self-efficacy. It assesses one's sense of personal competence and ability to complete tasks and reach goals, even when facing stressful situations (Bandura, 1977). The Italian version of the questionnaire has shown acceptable levels of reliability ( $\alpha=0.79$; Scholz et al., 2002). Cronbach's alpha for the current study was 0.88 .

\section{Anxiety and depression}

Anxiety and depression scores were assessed using the Hospital Anxiety and Depression Scale (HADS), a 14-item self-report scale (Zigmond and Snaith, 1983) comprised of an anxiety and a depression subscale. Higher scores indicate more depression and anxiety. The Italian version of the scale has shown good validity and high internal consistency ( $\alpha=0.89$; Costantini et al., 1999). Cronbach's alpha for the current sample ranged from $0.48-0.56$.

\section{Coping strategies}

Coping strategies were measured using the Brief-COPE (Carver, 1997), an instrument designed to evaluate how people handle stressful events. The Italian version of the scale has been validated (Conti, 1999) and includes 14 coping reactions: active coping, planning, positive reframing, acceptance, humor, religion, using emotional support, using instrumental support, self-distraction, denial, venting, substance use, behavioral disengagement, and self-blame. The alpha reliabilities of this 14 subscales ranged from 0.50 to 0.90 (Carver, 1997). In the present sample four scales (self-blame, venting, behavioral disengagement, substance use) showed unsatisfactory reliability with an alpha value lower than 0.50 , and were thus excluded from subsequent analyses. The alpha values of the remaining subscales ranged from 0.51 (for Active Coping) to 0.80 (for Religion).

\section{Adherence to medication}

Adherence to pharmacological treatment was measured by the Morisky Medical Adherence Scale-4 items (MMAS-4; Morisky et al., 1986). Higher scores indicate less adherence. The MMAS-4 showed moderate reliability $(\alpha=0.61)$ and good criterion-related, concurrent, and predictive validity (Morisky et al., 1986). In the present study Cronbach's alpha was 0.60.

\section{PROCEDURE}

Data were collected in the context of the ACTonHEART study, whose protocol was approved by the Istituto Auxologico Italiano Ethics Committee. Participants were recruited over a 16-month period by research staff at the beginning of the Cardiac Rehabilitation program. After giving permission to the use of their data for research purposes, participants completed a battery of measures including a demographics questionnaire, the HADS, the BRIEF-COPE, the CVD-AAQ item pool, the Morisky Adherence Scale, the GSE, the AAQ-II, and the PGWB in that order. A subgroup of participants $(n=132)$ also completed the PSS. Additional information such as weight, height and smoking status were self-reported by all participants. In order to evaluate test-retest reliability the CVD-AAQ was re-administered to 216 participants at one of three time points: after 7-14 days ( $n=57)$, after $21-28$ days $(n=110)$, and after $35-42$ days $(n=49)$.

\section{RESULTS \\ EXPLORATORY FACTOR ANALYSIS}

A exploratory factor analysis (EFA) was conducted on the initial 10 -item version of the questionnaire. The sampling adequacy for the analysis, verified by the Kaiser-Meyer-Olkin (KMO) index was very good for the total scale $(\mathrm{KMO}=0.81)$ and acceptable for all individual items $(\mathrm{KMO} \geq 0.57)$. Bartlett's test of sphericity indicated that correlations between items were sufficiently large for $\mathrm{EFA}\left[\chi^{2}(45)=592.66, p<0.0001\right]$.

An initial analysis was conducted with the principal axis factoring method with no restriction to the number of factors to be estimated. The initial run resulted in two factors with eigenvalues over Kaiser criterion of 1 (Kaiser, 1974), namely 3.22 and 1.56, explaining $47.86 \%$ of the total variance. The 
scree plot depicted a point of inflexion that would justify retaining two factors, with a third factor barely under 1 (0.942).

To better understand what model best represented the data, a parallel analysis (Horn, 1965) was computed. Previous research indicates that parallel analysis is a more accurate factor extraction procedure (Zwick and Velicer, 1986) than the scree plot or Kaiser's criterion. The results of the parallel analysis confirmed a twofactor model.

Since the two factors were expected to be elements of a single higher-order structure, namely psychological flexibility, an oblique rotation (Direct Oblimin) was applied (Nunnally, 1978). However, the percentage of variance explained by the two factors did not improve after rotation, and the correlation between the two factors remained very low $(r=0.04)$.

Moreover, reliability analyses indicated that items comprising the second factor (items 1,2,6) did not significantly correlate with the overall scale and their removal significantly increased Cronbach's alpha. An EFA of the remaining seven items was computed to evaluate the impact of item deletion to the scale's structure.

The scree plot depicted a point of inflection that suggested retaining one factor. This result was in line with the analysis of eigenvalues, with only one over Kaiser's criterion of 1 (3.12), explaining $44.55 \%$ of the variance. Thus, a one-factor solution was deemed appropriate, with a single factor thought to represent psychological inflexibility. In order to further justify a one-factor solution a second parallel analysis (Horn, 1965) was conducted. This additional analysis confirmed a one-factor solution for our measure. Table 2 shows the factor loadings from principal axis factoring of one and two-factor solutions, the latter without oblique rotation.

The internal consistency of the 7-item scale was found to be satisfactory, with a Cronbach's alpha of 0.79. All item-total correlations were above 0.50 except for item $4(r=0.36)$. These reliability indices were much improved from the 10 -item version, which showed a Cronbach's alpha of 0.67 and a lower item-total correlation (item 1, 2, and 6 below 0.30 ). Items 1, 2, and 6 were deleted and subsequent analyses utilized the 7-item version of the CVD-AAQ.

\section{TEST-RETEST RELIABILITY}

This section of the study was designed to examine test-retest reliability by calculating the two-way mixed, absolute concordance intraclass correlation coefficient (ICC). Of the 216 participants who completed the CVD-AAQ for a second time, a first subsample $(n=57)$ was retested after $7-14$ days, a second group $(n=110)$ after 21-28 days, and a third group $(n=49)$ after 35-42 days. The three groups did not significantly differ concerning demographics such as age $[F(2,216)=1.62, p=0.20]$, gender $\left[\chi^{2}(2)=0.32\right.$, $p=0.85]$, employment status $\left[\chi^{2}(6)=6.65, p=0.35\right]$, marital

\section{Table 2 | Factor loadings from principal axis factoring.}

\begin{tabular}{|c|c|c|c|}
\hline \multirow[t]{2}{*}{ CVD-AAQ item } & \multicolumn{2}{|c|}{ Two-factor solution } & \multirow{2}{*}{$\begin{array}{l}\text { One-factor } \\
\text { solution }\end{array}$} \\
\hline & Factor 1 & Factor 2 & \\
\hline 1. I try to avoid thinking about having heart disease & & 0.47 & l \\
\hline 2. I can lead a full and meaningful life even with heart disease ${ }^{a}$ & & 0.47 & / \\
\hline $\begin{array}{l}\text { 4. I eat things that are dangerous for my heart because the urge to } \\
\text { eat them is overwhelming }\end{array}$ & 0.39 & & 0.39 \\
\hline 5. Thinking about my heart disease is too or stressful for me & 0.61 & & 0.60 \\
\hline $\begin{array}{l}\text { 6. When I have an upsetting feeling or thought about my heart } \\
\text { disease I try to get rid of it }\end{array}$ & & 0.57 & l \\
\hline $\begin{array}{l}\text { 8. I do not exercise regularly because it reminds me that I have heart } \\
\text { disease }\end{array}$ & 0.64 & & 0.65 \\
\hline 9. I avoid thinking about the risks I face if I don't take care of my heart & 0.60 & & 0.60 \\
\hline $\begin{array}{l}\text { 10. I feel so scared by the thought of a possible relapse that I am not } \\
\text { able to commit myself to what really matters in my life }\end{array}$ & 0.67 & & 0.65 \\
\hline$\%$ of variance & 32.20 & 15.66 & 44.55 \\
\hline$\alpha$ & & & 0.79 \\
\hline
\end{tabular}

Only loadings above 0.3 are presented in the table.

a/tem reversed for scoring purposes. 
status $\left[\chi^{2}(8)=7.33, p=0.50\right]$, and education $\left[\chi^{2}(6)=3.38\right.$, $p=0.76]$. Moreover the three groups did not significantly differ concerning CVD-AAQ scores at time $1[F(2,216)=1.94$, $p=0.15]$.

Mean CVD-AAQ scores did not significantly change from the first to second administration for all three gathered together $[t(235)=0.19 ; p=0.85]$. Test-retest reliability appeared to decrease over time. According to Fleiss (1986), it was excellent after 7-14 days $(\mathrm{ICC}=0.90 ; \mathrm{CI}=0.83-0.94)$, good after $21-28$ days (ICC $=0.82 ;$ CI $=0.73-0.87)$, but only fair after 35-42 days $(\mathrm{ICC}=0.66 ; \mathrm{CI}=0.40-0.81)$.

\section{RELATIONSHIP WITH DEMOGRAPHICS}

A univariate analysis of variance (ANOVA) was conducted to explore the impact of gender, age, marital status, education, and employment status on psychological inflexibility related to cardiac disease.

As expected, there were no significant differences between CVD-AAQ scores in relation to gender $[F(1)=0.39, p=0.53]$, age $[F(1)=2.68, p=0.10]$, marital status $[F(4)=0.35, p=0.84]$, education $[F(3)=2.07, p=0.10]$ and employment status $[F(3)=1.98, p=0.12]$.

\section{VALIDITY}

\section{Construct validity}

In order to assess convergent validity a Pearson correlation coefficient was calculated between CVD-AAQ and AAQ-II. If the CVD-AAQ measures psychological inflexibility specific to the cardiovascular disease, it would be expected to be moderately correlated with measures of general psychological inflexibility. As shown in Table 3, the correlation between CVD-AAQ scores and AAQ-II $(r=0.50 ; p<0.001)$ supported the hypothesized relationship. Such correlation was stronger than the association with all the other measures, further supporting the construct validity of CVD-AAQ.

The associations of the CVD-AAQ with the different coping strategies assessed by the BRIEF-COPE were in line with our expectations. Cardiac-specific psychological inflexibility appeared to be significantly correlated with the "denial" subscale and to be inversely correlated with the "acceptance" scale. Although the association is weak, these results are consistent with the conceptual relatedness of these two scales with the experiential avoidance construct. Moreover, the remaining BRIEF-COPE scales did not show significant correlations with CVD-AAQ, further supporting the divergent validity of the instrument.

\section{Concurrent validity}

As expected, CVD-AAQ scores were significantly correlated with other psychological constructs of interest (see Table 3). In particular, higher levels of heart diseasespecific psychological inflexibility were significantly and moderately related to higher anxiety and depression (on both the HADS and the PGWB), higher stress (measured by the PSS), and to lower psychological well-being (on both the general health and vitality dimensions of PGWB). Moreover, a weak but significant correlation was found with self-efficacy (GSE).
Table 3 | Correlations between CVD-AAQ and other measures.

\begin{tabular}{|c|c|c|}
\hline & \multicolumn{2}{|c|}{ CVD-AAO } \\
\hline & $r$ & $p$ \\
\hline AAQ-II & 0.50 & $<0.001$ \\
\hline HADS sum score & 0.45 & $<0.001$ \\
\hline HADS anxiety & 0.44 & $<0.001$ \\
\hline HADS depression & 0.34 & $<0.001$ \\
\hline PGWB anxiety & 0.39 & $<0.001$ \\
\hline PGWB depressed mood & 0.45 & $<0.001$ \\
\hline PGWB self-control & -0.44 & $<0.001$ \\
\hline PGWB positive well-being & -0.35 & $<0.001$ \\
\hline PGWB general health & -0.37 & $<0.001$ \\
\hline PGWB vitality & -0.41 & $<0.001$ \\
\hline PGWB sum score & -0.48 & $<0.001$ \\
\hline PSS & 0.44 & $<0.001$ \\
\hline GSE & -0.25 & $<0.001$ \\
\hline Brief-Cope planning & -0.10 & 0.01 \\
\hline Brief-Cope self-distraction & 0.14 & 0.02 \\
\hline $\begin{array}{l}\text { Brief-Cope using } \\
\text { instrumental support }\end{array}$ & -0.02 & 0.72 \\
\hline $\begin{array}{l}\text { Brief-Cope using emotional } \\
\text { support }\end{array}$ & 0.13 & 0.04 \\
\hline $\begin{array}{l}\text { Brief-Cope positive } \\
\text { reframing }\end{array}$ & -0.06 & 0.30 \\
\hline Brief-Cope acceptance & -0.20 & 0.001 \\
\hline Brief-Cope religion & -0.07 & 0.26 \\
\hline Brief-Cope humor & -0.01 & 0.85 \\
\hline Brief-Cope denial & 0.22 & $<0.001$ \\
\hline
\end{tabular}

Positive correlations reflect higher levels of inflexibility and distress. AAQ-II, Acceptance and Action Questionnaire-ll; HADS, Hospital Anxiety and Depression Scale; PGWB, Psychological General Well Being; PSS, Perceived Stress Scale; GSE, General Self-Efficacy Scale.

Lastly, CVD-AAQ was significantly correlated with problems on adherence to medication $(r=0.27 ; p<0.001)$ and BMI $(r=0.23 ; p<0.001)$, while general psychological inflexibility, measured by the AAQ-II, reported a weaker association with the adherence measure $(r=0.15 ; p=0.01)$ and no correlation with BMI $(r=0.06 ; p=0.33)$.

\section{Independent sample t-test}

A two-tailed independent samples $t$-test showed that overweight participants (i.e., BMI > 25) had significantly higher CVDAAQ scores $(M=17.61, \mathrm{SD}=7.00)$, than those with a BMI below $25[M=15.00, \mathrm{SD}=5.90 ; t(272)=3.29, p<0.001]$ with a small effect size $(r=0.20)$. On the contrary, overweight $(M=19.53, \mathrm{SD}=8.50)$ and non-overweight $(M=18.31$, $\mathrm{SD}=7.50)$ patients did not significantly differ with respect to AAQ-II scores $[t(301)=1.30, p<0.19]$.

An independent samples $t$-test was then conducted to compare the CVD-AAQ score for patients with good adherence to 
medication (Morisky score $=0$ ) and patients with low adherence (Morisky score $\geq 1$ ). This latter category includes a wide range of patients with a suboptimal level of medication adherence ranging from occasionally missing a dose to missing the majority of medication doses. However, the above classification was considered clinically relevant, and was thus utilized for these analyses.

Patients with low adherence $(M=18.02, \mathrm{SD}=6.72)$ showed significantly higher scores on CVD-AAQ than patients with good medication adherence $[M=15.27, \mathrm{SD}=6.20 ; t(263)=3.34$, $p<0.001]$ with a small effect size $(r=0.19)$. This finding was not replicated with the AAQ-II; participants with low adherence, $(M=19.90, \mathrm{SD}=7.45)$ did not differ significantly on scores of general psychological inflexibility $[t(285)=-1.38, p<0.17]$ from those with good medication adherence $(M=18.51, \mathrm{SD}=8.38)$.

\section{DISCUSSION}

Although psychological inflexibility has been reported to play an important role in health-behavior change, there is currently no measure of this construct in the context of heart disease. Therefore, the CVD-AAQ was developed to fill the existing gap in the literature. The current study sought to investigate the psychometric characteristics of the CVD-AAQ in terms of factorial structure, reliability and validity.

The initial item pool was generated by applying the psychological inflexibility construct to the context of cardiovascular disease in order to measure the extent to which an individual makes cognitive and behavioral choices that are consistent with his/her values of health even when doing so lead to experiencing distressing thoughts or feelings. An EFA on this 10-item scale suggested a two-factor solution. However, reliability analyses showed that items loadings on the second factor did not significantly correlate with the overall scale and their deletion significantly increased Cronbach's alpha. After the items' removal, the resulting seven-item scale showed a 1-factor structure, a solution consistent with the AAQ-II. In conclusion, CVD-AAQ appears to measure the construct of psychological inflexibility with satisfactory internal consistency and test-retest reliability.

In addition to its sound factor structure and good reliability, our findings indicate that the CVD-AAQ is associated with variables to which it is theoretically tied. Specifically, the greatest association was observed with the AAQ-II, a general measure of the same construct, supporting the convergent validity of the instrument. On the other side, the absence of a significant correlation with all the coping strategies measured by the BRIEF-COPE, except for denial and acceptance, supported the divergent validity of the instrument.

Moreover, higher levels of cardiac-specific psychological inflexibility were concurrently associated with greater depressive symptoms, anxiety, and psychological stress and lower levels of psychological well-being. These results are highly consistent with previous research on general psychological inflexibility, which appeared to be a key process in the etiology and maintenance of psychopathology and psychological suffering (Hayes et al., 2006; Gaudiano, 2011).

Consistent with the existing literature (Bond et al., 2011), psychological inflexibility was not associated with demographic variables. Cardiac-specific psychological inflexibility was greater in patients with low rates of adherence to medical treatmentfindings that mirror previous research on disease-specific psychological inflexibility (Weijman et al., 2005). This finding suggests that psychological flexibility may be associated with the adoption and maintenance of health behaviors, such as medication adherence, among patients with heart disease.

Cardiovascular Disease Acceptance and Action Questionnaire yields substantially different scores among cardiac patients with a BMI $>25$ compared with those with a BMI below 25 . This finding indicates that the overweight patients are more psychologically inflexible and suggests that difficulty in weight management may be associated with the individual's tendency to sacrifice long-term behavioral goals (e.g., healthy food choices and exercise) in the service of decreasing unpleasant and distressful internal experiences (e.g., food cravings and fatigue).

Moreover, this result is of great importance because BMI and adherence to medication are two relevant risk factors for cardiovascular disease. Notably, no between-group differences were detected using the AAQ-II, relative to both BMI and adherence. This result speaks to the incremental validity of CVD-AAQ and supports the usefulness of disease- and behavior-specific instruments for assessing psychological inflexibility.

Previous research suggests that psychological inflexibility plays an important role in the adoption and maintenance of health behaviors. Moreover, a preliminary investigation by Goodwin et al. (2012) found that a program to enhance psychological flexibility in a sample of cardiac patients effectively improved adherence to heart-healthy behaviors.

The current study is the first to evaluate the usefulness of a measure of psychological inflexibility specific to cardiovascular disease. The use of the CVD-AAQ may help identify patients who are more likely to be non-adherent. With further evaluation of the CVD-AAQ, it is possible that use of the instrument could help identify patients who are at risk for relapse or re-events. In an effort to begin such an evaluation, the CVD-AAQ will be employed to evaluate the mediational role of psychological flexibility in the context of a RCT comparing a brief ACT-based intervention to usual secondary prevention care of coronary heart disease (Spatola et al., 2014).

However, there are several limitations to the current study and further investigation is needed before this measure can be confidently used to assess the constructs of psychological inflexibility. Firstly, further tests of reliability and validity in other countries would ensure that the psychometric properties of this measure are well established and replicable. We considered running a confirmatory factor analysis in order to examine method variance, but since the initial one-factor solution which led to the development of the instrument was based on the same sample, we were concerned that any findings might be due to capitalization on chance, rather than true variance. Thus, we decided to not run this analysis until another sample could be obtained. Our recommendation is that future studies of the CVD-AAQ with other samples include a confirmatory factor analysis that allows for examination of whether the single factor solution found in our EFA is a better fit for the data than a two-factor solution. Secondly, the inclusion of a broader range of measures would 
be of particular utility for a deeper understanding of CVD-AAQ validity, with particular attention to be paid to other psychological inflexibility measures such as the Cognitive Fusion Questionnaire (Gillanders et al., 2014). Finally, longitudinal studies are needed in order to clarify the association between the CVD-specific psychological inflexibility and patient ability to adopt or maintain a heart-healthy lifestyle.

\section{REFERENCES}

Bandura, A. (1977). Self-efficacy: toward a unifying theory of behavioral change. Psychol. Rev. 84, 191-215. doi: 10.1037/0033-295X.84.2.191

Bond, F. W., Hayes, S. C., Baer, R. A., Carpenter, K. M., Guenole, N., Orcutt, H. K., et al. (2011). Preliminary psychometric properties of the acceptance and action questionnaire-II: a revised measure of psychological inflexibility and experiential avoidance. Behav. Ther. 42, 676-688. doi: 10.1016/j.beth.2011. 03.007

Brown, R. A., Lejuez, C. W., Kahler, C. W., Strong, D. R., and Zvolensky, M. J. (2005). Distress tolerance and early smoking lapse. Clin. Psychol. Rev. 25, 713-733. doi: 10.1016/j.cpr.2005.05.003

Butryn, M. L., Arigo, D., Raggio, G. A., Kaufman, A. I., Kerrigan, S. G., and Forman, E. M. (2014). Measuring the ability to tolerate activity-related discomfort: initial validation of the physical activity acceptance questionnaire (PAAQ). J. Phys. Act. Health doi: 10.1123/jpah.2013-0338 [Epub ahead of print].

Butryn, M. L., Forman, E., Hoffman, K., Shaw, J., and Juarascio, A. (2011). A pilot study of acceptance and commitment therapy for promotion of physical activity. J. Phys. Act. Health 8, 516-522.

Carver, C. S. (1997). You want to measure coping but your protocol's too long: consider the brief COPE. Int. J. Behav. Med. 4, 92-100. doi: 10.1207/s15327558ijbm0401_6

Cohen, S., and Janicki-Deverts, D. (2012). Who's stressed? distributions of psychological stress in the united states in probability samples from 1983, 2006 and 2009. J. Appl. Soc. Psychol. 42, 1320-1334. doi: 10.1111/j.1559-1816.2012.00900.x

Cohen, S., Kamarck, T., and Mermelstein, R. (1983). A global measure of perceived stress. J. Health Soc. Behav. 24, 385-396. doi: 10.2307/2136404

Conti, L. (1999). Repertorio Delle Scale Di Valutazione in Psichiatria. Firenze: Edizioni SEE.

Costantini, M., Musso, M., Viterbori, P., Bonci, F., Del Mastro, L., Garrone, O., et al. (1999). Detecting psychological distress in cancer patients: validity of the italian version of the hospital anxiety and depression scale. Support. Care Cancer 7, 121-127. doi: 10.1007/s005200050241

Dimsdale, J. E. (2008). Psychological stress and cardiovascular disease. J. Am. Coll. Cardiol. 51, 1237-1246. doi: 10.1016/j.jacc.2007.12.024

Dorneleas, E. (2008). Psychotherapy with Cardiac Patients: Behavioral Cardiology in Practice. Washington, DC: American Psychological Association. doi: 10.1037/11809-000

Dupuy, H. J. (1984). "The psychological general well-being (PGWB) index," in Assessment of Quality of Life in Clinical Trials of Cardiovascular Therapies, eds N. K. Wenger, M. E. Mattson, C. D. Furberg and J. Elinson (New York, NY: Le Jacq), $170-183$.

Fish, R. A., McGuire, B., Hogan, M., Morrison, T. G., and Stewart, I. (2010). Validation of the chronic pain acceptance questionnaire (CPAQ) in an internet sample and development and preliminary validation of the CPAQ-8. Pain 149, 435-443. doi: 10.1016/j.pain.2009.12.016

Fleiss, J. (1986). The Design and Analysis of Clinical Experiments. New York, NY: John Wiley and Sons.

Forman, E. M., Butryn, M. L., Hoffman, K., and Herbert, J. D. (2009). An open trial of an acceptance-based behavioral treatment for weight loss. Cogn. Behav. Pract. 16, 223-235. doi: 10.1016/j.cbpra.2008.09.005

Forman, E. M., Hoffman, K. L., McGrath, K. B., Herbert, J. D., Brandsma, L. L., and Lowe, M. R. (2007). A comparison of acceptance- and control-based strategies for coping with food cravings: an analog study. Behav. Res. Ther. 45, 2372-2386. doi: 10.1016/j.brat.2007.04.004

Gaudiano, B. A. (2011). Evaluating acceptance and commitment therapy: an analysis of a recent critique. Int. J. Behav. Consult. Ther. 7, 55-67.

Gifford, E., Kohlenberg, B., Hayes, S. C., Antonuccio, D., Piasecki, M., and Rasmussen-Hall, L. (2004). Acceptance-based treatment for smoking cessation. Behav. Ther. 35, 689-705. doi: 10.1016/S0005-7894(04)80015-7
Gillanders, D. T., Bolderston, H., Bond, F. W., Dempster, M., Flaxman, P. E., Campbell, L., et al. (2014). The development and initial validation of the cognitive fusion questionnaire. Behav. Ther. 45, 83-101. doi: 10.1016/j.beth.2013.09.001

Goodwin, C. L., Forman, E. M., Herbert, J. D., Butryn, M. L., and Ledley, G. S. (2012). A pilot study examining the initial effectiveness of a brief acceptancebased behavior therapy for modifying diet and physical activity among cardiac patients. Behav. Modif. 36, 199-217. doi: 10.1177/0145445511427770

Gregg, J. A., Callaghan, G. M., Hayes, S. C., and Glenn-Lawson, J. L. (2007). Improving diabetes self-management through acceptance, mindfulness, and values: a randomized controlled trial. J. Consult. Clin. Psychol. 75, 336-343. doi: 10.1037/0022-006X.75.2.336

Grossi, E., Mosconi, P., Groth, N., Niero, M., and Apolone, G. (2002). Il Questionario Psychological General Well Being. Questionario Per la Valutazione Dello Stato Generale di Benessere Psicologico, Versione Italiana. Milano: Istituto di ricerche farmacologiche "Mario Negri."

Hayes, S. C., Follette, V. M., and Linehan, M. M. (2004). Mindfulness and Acceptance: Expanding the Cognitive-Behavioral Tradition. New York, NY: Guilford.

Hayes, S. C., Luoma, J. B., Bond, F. W., Masuda, A., and Lillis, J. (2006). Acceptance and commitment therapy: model, processes and outcomes. Behav. Res. Ther. 44, 1-25. doi: 10.1016/j.brat.2005.06.006

Hayes, S. C., Strosahl, K., and Wilson, K. G. (1999). Acceptance and Commitment Therapy: An Experiential Approach to Behavior Change. New York, NY: Guilford.

Ho, P. M., Magid, D. J., Shetterly, S. M., Olson, K. L., Maddox, T. M., Peterson, P. N., et al. (2008). Medication nonadherence is associated with a broad range of adverse outcomes in patients with coronary artery disease. Am. Heart J. 155, 772-779. doi: 10.1016/j.ahj.2007.12.011

Horn, J. L. (1965). A rationale and test for the number of factors in factor analysis. Psychometrika 30, 179-185. doi: 10.1007/BF02289447

Januzzi, J. L. Jr., Stern, T. A., Pasternak, R. C., and DeSanctis, R. W. (2000). The influence of anxiety and depression on outcomes of patients with coronary artery disease. Arch. Intern. Med. 160, 1913-1921. doi: 10.1001/archinte.160.13.1913

Juarascio, A., Forman, E., Timko, C. A., Butryn, M., and Goodwin, C. (2011). The development and validation of the food craving acceptance and action questionnaire (FAAQ). Eat. Behav. 12, 182-187. doi: 10.1016/j.eatbeh.2011.04.008

Kaiser, H. F. (1974). An index of factorial simplicity. Psychometrika 39, 31-36. doi: 10.1007/BF02291575

Lillis, J., Hayes, S. C., Bunting, K., and Masuda, A. (2009). Teaching acceptance and mindfulness to improve the lives of the obese: a preliminary test of a theoretical model. Ann. Behav. Med. 37, 58-69. doi: 10.1007/s12160-0099083-x

Lundgren, T., Dahl, J., and Hayes, S. C. (2008). Evaluation of mediators of change in the treatment of epilepsy with acceptance and commitment therapy. J. Behav. Med. 31, 225-235. doi: 10.1007/s10865-008-9151-x

Luoma, J., Drake, C. E., Kohlenberg, B. S., and Hayes, S. C. (2011). Substance abuse and psychological flexibility: the development of a new measure. Addict. Res. Theory 19, 3-13. doi: 10.3109/16066359.2010.524956

Morisky, D. E., Green, L. W., and Levine, D. M. (1986). Concurrent and predictive validity of a self-reported measure of medication adherence. Med. Care 24, 67-74. doi: 10.1097/00005650-198601000-00007

Nunnally, J. (1978). Psychometric Theory, 2nd Edn. New York, NY: McGraw-Hill.

Pennato, T., Berrocal, C., Bernini, O., and Rivas, T. (2013). Italian version of the acceptance and action questionnaire-II (AAQ-II): dimensionality, reliability, convergent and criterion validity. J. Psychopathol. Behav. Assess. 35, 552-563. doi: 10.1007/s10862-013-9355-4

Rothenbacher, D., Jaensch, A., Mons, U., Hahmann, H., Becker, T., Koenig, W., et al. (2014). Prognostic value of one-year course of symptoms of anxiety and depression in patients with coronary heartdisease: role of physical activity and unmet medical need. Eur. J. Prev. Cardiol. doi: 10.1177/2047487314545317 [Epub ahead of print].

Rozanski, A., Blumenthal, J. A., Davidson, K. W., Saab, P. G., and Kubzansky, L. (2005). The epidemiology, pathophysiology, and management of psychosocial risk factors in cardiac practice: the emerging field of behavioral cardiology. J. Am. Coll. Cardiol. 45, 637-651. doi: 10.1016/j.jacc.2004.12.005

Sandoz, E. K., Wilson, K. G., Merwin, R. M., and Kellum, K. K. (2013). Assessment of body image flexibility: the body image-acceptance and action questionnaire $J$. Contextual Behav. Sci. 2, 39-48. doi: 10.1016/j.jcbs.2013.03.002

Scholz, U., Gutiérrez Doña, B., Sud, S., and Schwarzer, R. (2002). Is general selfefficacy a universal construct? Psychometric findings from 25 countries. Eur. J. Psychol. Assess. 18, 242-251. doi: 10.1027//1015-5759.18.3.242 
Schwarzer, R., and Jerusalem, M. (1995). "Generalized self-efficacy scale," in Measures in Health Psychology: A User's Portfolio. Causal and Control Beliefs, eds J. Weinman, S. Wright, and M. Johnston (Windsor: NFER-NELSON), 35-37.

Spatola, C. A., Manzoni, G. M., Castelnuovo, G., Malfatto, G., Facchini, M., Goodwin, C. L., etal. (2014). The ACTonHEART study: rationale and design of a randomized controlled clinical trial comparing a brief intervention based on acceptance and commitment therapy to usual secondary prevention care of coronary heart disease. Health Qual. Life Outcomes 12, 22. doi: 10.1186/1477-7525-12-22

Strike, P. C., and Steptoe, A. (2004). Psychosocial factors in the development of coronary artery disease. Prog. Cardiovasc. Dis. 46, 337-347. doi: 10.1016/j.pcad.2003.09.001

Weijman, I., Ros, W. J., Rutten, G. E., Schaufeli, W. B., Schabracq, M. J., and Winnubst, J. A. (2005). The role of work-related and personal factors in diabetes self-management. Patient Educ. Couns. 59, 87-96. doi: 10.1016/j.pec.2004.10.004

Westin, V., Hayes, S. C., and Andersson, G. (2008). Is it the sound or your relationship to it? The role of acceptance in predicting tinnitus impact. Behav. Res. Ther. 46 , 1259-1265. doi: 10.1016/j.brat.2008.08.08

Yusuf, S., Hawken, S., Ounpuu, S., Dans, T., Avezum, A., Lanas, F., et al. (2004). Effect of potentially modifiable risk factors associated with myocardial infarction in 52 countries (the INTERHEART study): case-control study. Lancet 364, 937-952. doi: 10.1016/S0140-6736(04)17018-9

Zigmond, A. S., and Snaith, R. P. (1983). The hospital anxiety and depression scale. Acta Psychiatr. Scand. 67, 361-370. doi: 10.1111/j.1600-0447.1983.tb09716.x
Zwick, W. R., and Velicer, W. F. (1986). Comparison of five rules for determining the number of components to retain. Psychol. Bull. 99, 432-442. doi: 10.1037/00332909.99.3.432

Conflict of Interest Statement: The authors declare that the research was conducted in the absence of any commercial or financial relationships that could be construed as a potential conflict of interest.

Received: 01 August 2014; accepted: 23 October 2014; published online: 14 November 2014.

Citation: Spatola CAM, Cappella EAM, Goodwin CL, Baruffi M, Malfatto G, Facchini M, Castelnuovo G, Manzoni GM and Molinari E (2014) Development and initial validation of the Cardiovascular Disease Acceptance and Action Questionnaire (CVD-AAQ) in an Italian sample of cardiac patients. Front. Psychol. 5:1284. doi: 10.3389/fpsyg.2014.01284

This article was submitted to Psychology for Clinical Settings, a section of the journal Frontiers in Psychology.

Copyright (c) 2014 Spatola, Cappella, Goodwin, Baruffi, Malfatto, Facchini, Castelnuovo, Manzoni and Molinari. This is an open-access article distributed under the terms of the Creative Commons Attribution License (CC BY). The use, distribution or reproduction in other forums is permitted, provided the original author(s) or licensor are credited and that the original publication in this journal is cited, in accordance with accepted academic practice. No use, distribution or reproduction is permitted which does not comply with these terms. 